\title{
Dissipative soliton resonances in laser models with parameter management
}

\author{
Wonkeun Chang, ${ }^{1, *}$ Adrian Ankiewicz, ${ }^{1}$ Jose M. Soto-Crespo, $^{2}$ and N. Akhmediev ${ }^{1}$ \\ ${ }^{1}$ Optical Sciences Group, Research School of Physical Sciences and Engineering, The Australian National \\ University, Canberra ACT 0200, Australia \\ ${ }^{2}$ Instituto de Óptica, C.S.I.C., Serrano 121, 28006 Madrid, Spain \\ *Corresponding author: wonkeun.chang@anu.edu.au
}

Received July 29, 2008; revised October 3, 2008; accepted October 6, 2008;

posted October 9, 2008 (Doc. ID 99474); published November 13, 2008

\begin{abstract}
Dissipative soliton resonance (DSR) is a phenomenon where the energy of a soliton in a dissipative system increases without limit at certain values of the system parameters. We have found that the DSR phenomenon is robust and does not disappear when perturbations are introduced into the model. In particular, parameter management is benign to DSR: the resonance property remains intact even when a pulse experiences periodic changes of system parameters in a laser cavity. We also show that high energy pulses emerging from a laser cavity can be compressed to shorter durations with the help of linear dispersive devices. (C) 2008 Optical Society of America
\end{abstract}

OCIS codes: $190.4370,320.5520$.

\section{INTRODUCTION}

Dissipative soliton resonance (DSR) is a phenomenon where the energy of a soliton in a dissipative system becomes infinitely high at certain values of the system parameters. Specifically, we have found that systems that can be modeled using the cubic-quintic complex Ginzburg-Landau equation (CGLE)[1,2] admit a region of parameters with stable solitons whose energy goes to infinity at the boundary of that region. The edge of this region in our five-dimensional parameter space is a codimension one surface. Thus, the energy of the dissipative solitons remains infinite even when the parameters are changed in a continuous way along that surface. Clearly, the definition of 'resonance' used here is wider than that used in the physics of linear oscillations. Instead of varying frequencies, we vary the generalized system parameters to achieve the emission of high energy pulses. This phenomenon can be useful in designing optical oscillators generating pulses with exceptionally high energies [3].

Until now, there has not been a way to find the DSR using any analytical procedure. However, we can use trial function techniques to find the resonance approximately [4]. The method allows us to obtain an equation that roughly describes the position of the resonant surface in the multidimensional parameter space. Numerical simulations of the CGLE allow us to confirm their existence and to find the resonant surface more accurately. A question arises as to whether the resonance is a robust phenomenon relative to perturbations of the model itself. To be specific, does the phenomenon still exist when we make the model closer to real systems? The CGLE is a good approximation for describing optical oscillators with passive mode-locking [5]. However, a better approximation is needed in specific cases.

In this paper, we answer this question by introducing parameter management into the system. Thus, we allow for changes in the medium in which the pulse propagates, and this means that the parameters of the governing equation also change. If we are dealing with a fiber laser $[6,7]$, then typically the pulse periodically passes through an erbium-doped fiber, a mode-locking device, and a standard single mode fiber. Passing through the amplifier and mode-locking element in the cavity can be described by the nonlinear nonconservative terms of the master equation while the propagation through the single mode fiber is affected simply by second-order dispersion. In solidstate lasers, such as Ti:sapphire lasers with Kerr-lens mode-locking [8], prisms and mirrors provide purely chromatic dispersion while the crystal itself serves simultaneously as an amplifier and nonlinear transmission element. Thus, a periodic change in the parameters of the CGLE will roughly describe the propagation of the pulse in laser cavities [9].

These modifications are certainly needed if the pulse experiences relatively large changes during each round trip around the cavity. If the pulse variations are modest, then the averaged model [10] with constant parameters is good enough to describe the pulse generation process. Thus, to be even more specific, our task in this paper is to answer the question outlined above: will the periodicity in the cavity result in the disappearance of the DSR, or is the phenomenon so robust that these changes will influence only the location of the resonance surface but not cause the phenomenon to fade? For this purpose, we introduce a model that does not describe any specific laser system but does allow for relatively large changes in the pulse evolution. In numerical modeling of this particular situation, we have found that the DSR phenomenon does indeed persist. It does not disappear and we can hope that a real system can be suitably constructed to produce high 
energy pulses. The system dispersion generally has to be chosen in the normal regime [6], but this is not an absolutely necessary condition.

\section{MODEL}

The sketch of the laser model that we consider in this paper is shown in Fig. 1(a). It consists of three main elements. (1) A single-mode fiber, of length $L_{2}$, where only second-order chromatic dispersion takes place. (2) A piece of fiber with length $L_{1}$, where amplification, nonlinearity, and mode-locking occur. (3) An 80/20 coupler located in the middle of the element of length $L_{1}$. Another example that is described qualitatively by this model would be a solid-state laser with Kerr-lens mode-locking [8,11]. In the latter case, the mode-locking and amplification are concentrated inside the laser crystal, and the rest of the cavity can be described as a dispersive line. The model is very simple and serves only for the demonstration of the principle. If the DSR does exist in this model, there is no reason why it should disappear in more complicated systems.

Pulse propagation in a passively mode-locked laser can be modeled by the CGLE. This is given by [2]

$$
i \psi_{z}+\frac{D}{2} \psi_{t t}+|\psi|^{2} \psi+\nu|\psi|^{4} \psi=i \delta \psi+i \epsilon|\psi|^{2} \psi+i \beta \psi_{t t}+i \mu|\psi|^{4} \psi
$$

where $z$ is the propagation distance normalized to the cavity round trip length, $t$ is the retarded time, $\psi$ is the normalized envelope of the field, $D$ is the group velocity dispersion coefficient being positive (negative) in the anomalous (normal) dispersion regime, $\delta$ is the linear

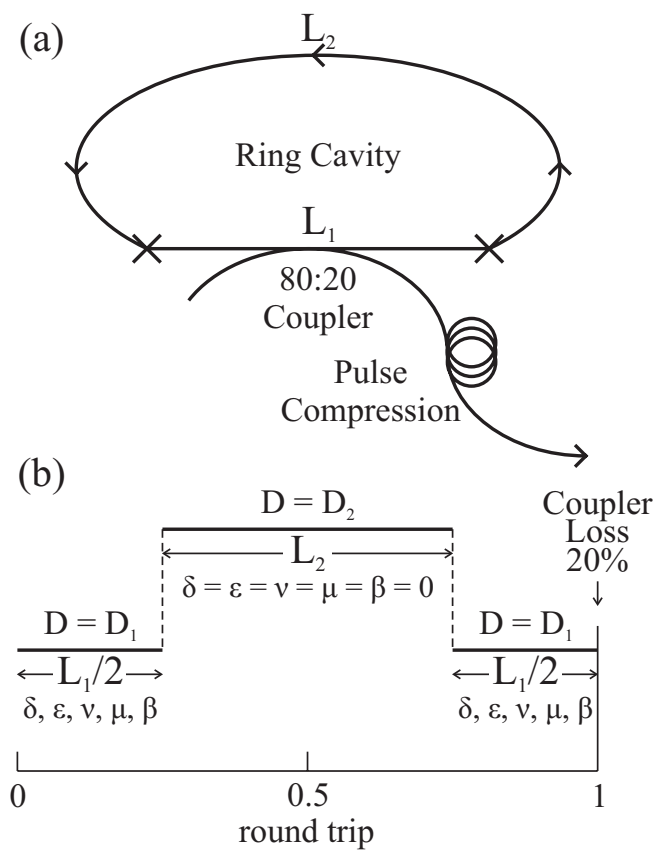

Fig. 1. (a) Schematic of the model and (b) map of the parameters of the CGLE for a single round trip of the pulse in the cavity. Pulses travel counterclockwise around the nonlinear $\left(L_{1}\right)$ and linear $\left(L_{2}\right)$ sections of the loop. At the coupler, $20 \%$ of the light is tapped off, and this enters the compressing piece of fiber.

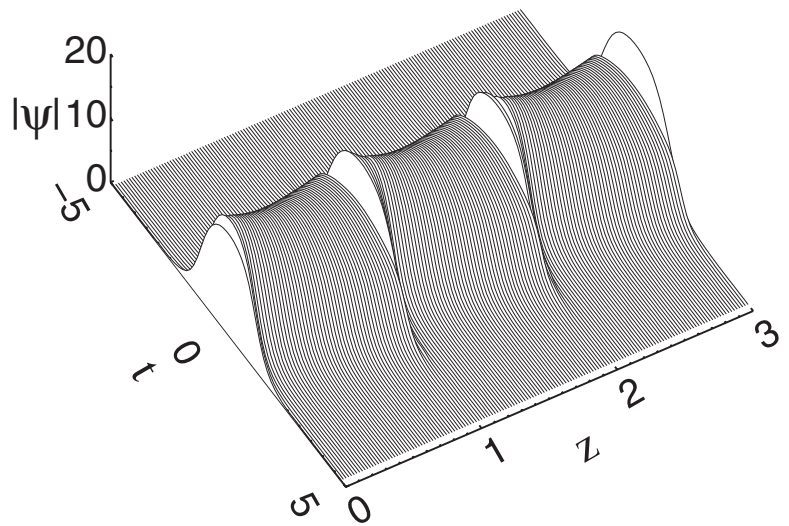

Fig. 2. Evolution of the soliton within the dispersion-managed cavity for three consecutive round trips. The pulse shape repeats at the end of each round trip, and hence fixed shape solitons are generated at the output of the oscillator. The system parameters are $L_{1}=0.1, L_{2} D_{2}=-0.02, D_{1}=-1, \epsilon=1.0, \delta=1.1, \beta=0.08, \nu=0.01$, and $\mu=-0.003$.

gain-loss coefficient, $\beta$ accounts for spectral filtering and $\epsilon$ represents the nonlinear gain; the term with $\mu$ represents, if negative, the saturation of the nonlinear gain, while the one with $\nu$ corresponds to the quintic nonlinearity.

The propagation of a pulse through a laser cavity is a periodic process [9] that can be modeled by Eq. (1), but here the parameters $D, \delta, \beta, \epsilon, \mu$, and $\nu$ are periodic functions of $z$. The period is defined by the cavity round trip, and the parameters are stepwise functions of $z$. The parameter map that we are using in this paper is shown in Fig. 1(b). Clearly, there is a great variety of laser designs [12-16], and this particular map cannot cover all of them. However, as we mentioned, it serves as an example, which shows that the model admits the resonance phenomenon.

In our model, amplification and nonlinear effects are concentrated in a section of the cavity of length $L_{1}$ while a section of length $L_{2}$ (standard telecom fiber or prisms and mirrors) serves as the dispersive line. Equation (1) in Section 1 is solved using a split-step Fourier method. In Sec-

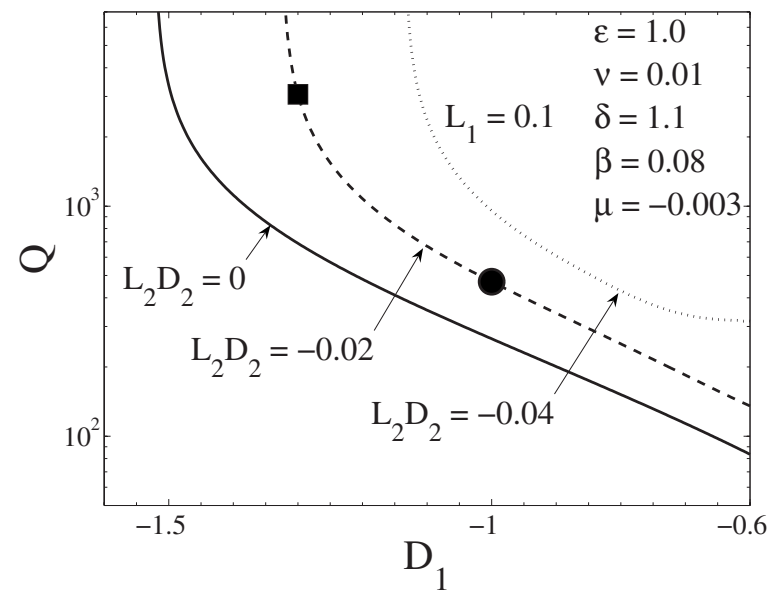

Fig. 3. Soliton energy, $Q$, in the $L_{1}$ section of the oscillator versus the dispersion parameter, $D_{1}$, for three different values of $L_{2} D_{2}$. The solid circle and the solid square show the points for which pulses are compressed (see Fig. 6 below). 
tion 2 , the dispersion is the only effect that acts on the pulse. Here, Eq. (1) can be reduced to its simpler version

$$
i \psi_{z}+\frac{D_{2}}{2} \psi_{t t}=0
$$

A stationary regime of pulse evolution in the cavity is shown in Fig. 2. The stationary regime is reached after a few round trips, provided that the system parameters are chosen in the range of stable soliton solutions. The cavity parameters that we have used are given in the Fig. 2 caption. The pulse evolution is periodic, with the width increasing in the dispersive part of the cavity and the amplitude increasing in the part of the cavity with amplification. Within the fiber loop, the pulse energy drops by $20 \%$ each time the pulse passes the coupler. This implies that small amplitude waves experience an overall linear loss, despite the fact that $\delta$ is positive in the active medium. The pulse energy, $Q=\int_{-\infty}^{\infty}|\psi|^{2} \mathrm{~d} t$, is monitored at the end of each round trip, i.e., at point 1 in Fig. 1. The pulses outside the cavity, i.e., in the lower coupler output, have an energy that is $20 \%$ of the value of $Q$ before it.

\section{RESONANCE}

Figure 3 shows the pulse energy, $Q$, at point 1 of the cavity, i.e., before the pulse passes the coupler, versus the dispersion parameter, $D_{1}$. The three distinct curves in this plot are calculated for three values of the linear disper- sion, $L_{2} D_{2}$. The case $L_{2} D_{2}=0$ corresponds to the case without the purely dispersive piece. Thus, the pulse propagation is described by a single CGLE with constant coefficients, and it is disturbed only by the coupler. This case is the closest to the model that was studied before in $[3,4]$.

The major feature of the plot in Fig. 3 is that the energy, $Q$, increases to infinitely large values at the lefthand side of each curve. This happens for every value of the linear dispersion, $L_{2} D_{2}$. This is exactly the same feature that was observed in the continuous model [3]. We called this phenomenon "dissipative soliton resonance" [4]. As we can see, the phenomenon does not disappear when we move away from the continuous model. Thus, the resonance of dissipative solitons is a robust phenomenon that reveals itself even when the model is perturbed.

The pulse profiles and the corresponding spectra are shown in Figs. 4(a) and 4(b), respectively, for three different values of $D_{1}$. The pulse width in Fig. 4(a) is the highest for $D_{1}=-1.3$. When the dispersion, $D_{1}$, approaches the point of resonance, the width of the soliton further increases without limit. The spectra are quite distinctive when the cavity is in the negative dispersion regime. They are symmetric and have two side maxima. The strength of the central maximum in Fig. 4(b) increases when we approach the point of resonance. This is also in accordance with the fact that the soliton energy increases to infinity. Although the spectra of the pulses are not ex-
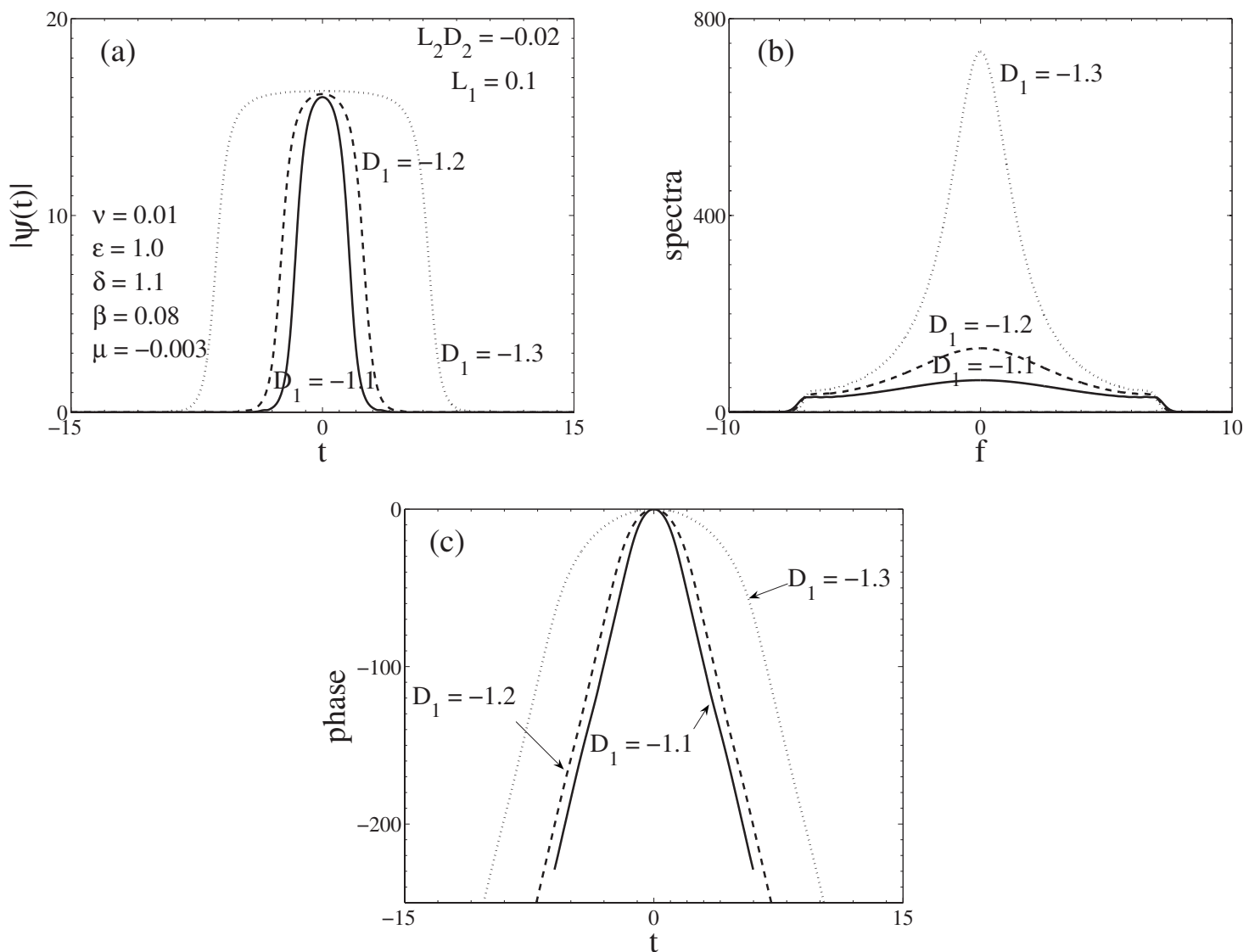

Fig. 4. (a) Pulse profiles, (b) soliton spectra, and (c) phase profiles for three different values of $D_{1}$. The rest of the parameters are shown inside (a). 

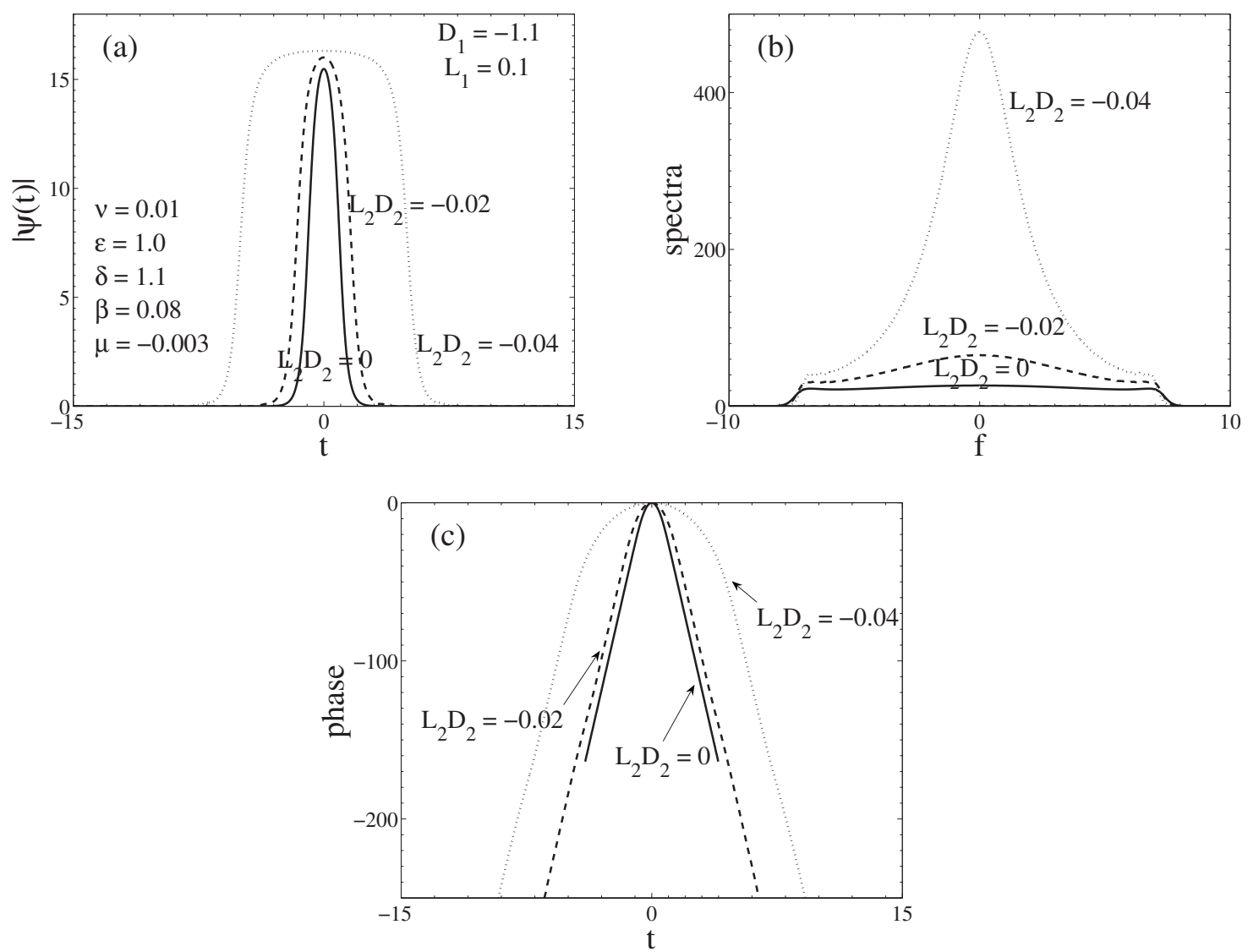

Fig. 5. (a) Pulse profiles, (b) soliton spectra, and (c) phase for three different values of $L_{2} D_{2}$. The rest of the parameters are shown inside (a). Comparison with Fig. 4 shows that the effect of changing $L_{2} D_{2}$ is qualitatively the same as the effect of changing $D_{1}$.

actly the same as in the continuous model [3], their changes when approaching the resonance follow the same scenario.

When we change $L_{2} D_{2}$ to negative values, the energy, $Q$, also increases, as can be seen in Fig. 3. Figures 5(a) and 5(b) show the change in the pulse profiles and their spectra in this case. A comparison with the data in Fig. 4 shows that changing $L_{2} D_{2}$ has qualitatively the same effect as changing the linear dispersion $D_{1}$.

Moreover, the curves in Fig. 3 have fairly similar shapes, and so we can also conclude from this that

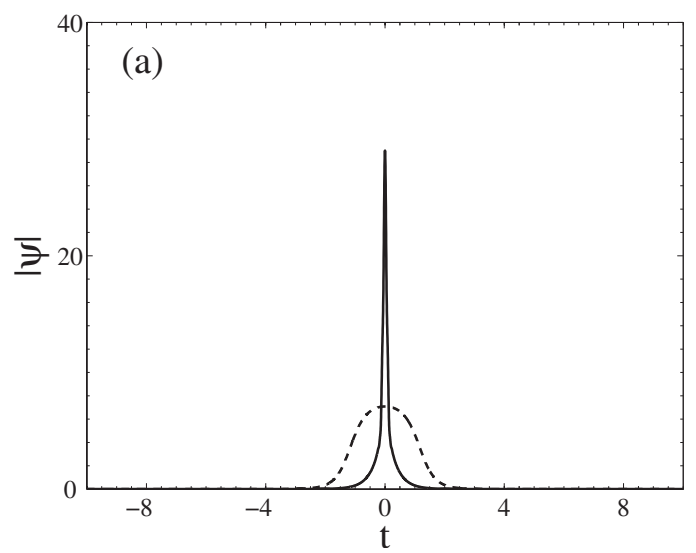

changes in $D_{1}$ and $D_{2}$ may have similar effects. In fact, the three curves in Fig. 3 can be roughly approximated by the following empirical expression:

$$
\log _{10}(Q)=\frac{2.8}{\langle D\rangle+2.2}
$$

where the effective (average) dispersion is defined by

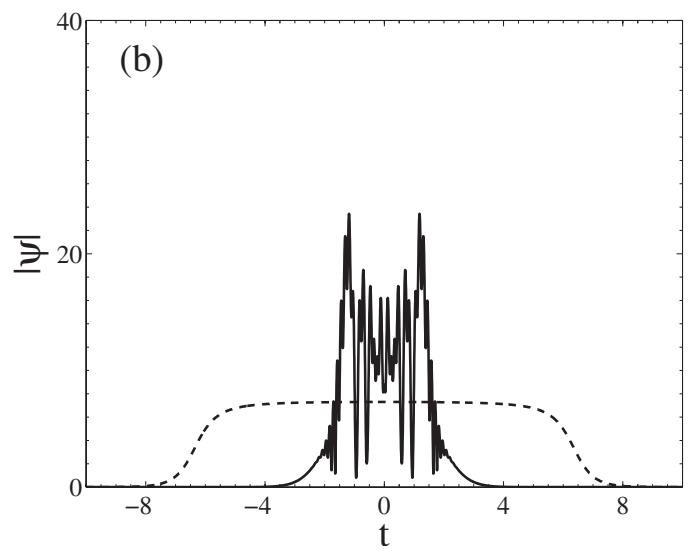

Fig. 6. Pulse profiles before (dashed curves) and after (solid curves) the linear compression of the pulse. The oscillator output pulses (dashed curves) are chosen for the set of parameters shown in Fig. 3 and (a) $D_{1}=-1.0$ (solid circle in Fig. 3 and (b) $D_{1}=-1.3$ (solid square in Fig. 3). 


$$
\langle D\rangle=D_{1}+10 L_{2} D_{2}
$$

Therefore, we can represent the results in Fig. 3 by using the $L_{2} D_{2}=0$ curve if we replace $D_{1}$ with $\langle D\rangle$ on the horizontal axis. Thus, the system functions like a single element with an effective value of dispersion. However, this is only an approximation.

\section{PULSE COMPRESSION}

Indeed, the energy of the pulse outputs generated from the oscillator that we have discussed in Section 3 increases dramatically as we approach the resonance. This increase is achieved mainly from the increase in the pulse width rather than from an increase in its amplitude. The peak amplitude is usually limited by the parameters of the mode-locking device, i.e., by the saturation of the nonlinear transmission (in our model basically by $\epsilon$ and $\mu$ ). Nevertheless, as the output pulse from the oscillator is highly chirped, this can be used to compress the pulse [17] and hence obtain high energy ultrashort pulses.

The phase profiles of the output pulses are shown in Fig. 4(c) and Fig. 5(c). The phase is roughly parabolic in the central part of the pulse and linear in the soliton wings. Each pulse is very far from being transformlimited. The presence of the chirp makes it possible to carry out pulse compression using linear devices [17]. One of the simplest methods of compressing such pulses is to use a linearly dispersive medium, such as a pair of gratings [18] or a dispersive fiber, to compensate for the linear component of the chirp. A compression scheme is known to work well for a linearly chirped pulse [19]. If the initial pulse is Gaussian, then its shape is retained after compression. In our case, the pulse is not Gaussian and the chirp is not linear across the whole pulse but, nonetheless, a good compression can still be achieved.

Mathematically speaking, compression is achieved with the use of Eq. (2), where $D_{2}$ is chosen to be positive. The particular value of $D_{2}$ influences only the length of propagation distance needed for achieving the optimal compression. Figure 6 shows the results for the numerical pulse compression of two different pulses taken at the output of the oscillator (point 1 in Fig. 1). When the parameters of the oscillator are chosen to be not too close to the resonance point, the pulse can be compressed nicely to a fraction of its initial width. The resulting pulse shape is close to being bell-shaped with a single maximum. The compression result for the solid black point in Fig. 1 is shown in Fig. 6(a). The dashed curve in Fig. 6 shows the pulse shape before compression, and the solid curve corresponds to the pulse shape after the compression.

When the parameters are chosen closer to the resonance point, the chirp has a large part that is far from being linear. Then linear compression does not completely eliminate the chirp. The pulse is compressed but not up to the transform limit. The compression result for the black solid square point in Fig. 1 is shown in Fig. 6(b). The dashed curve shows the pulse shape out of the oscillator while the solid curve corresponds to the compressed pulse. The remaining chirp leads to the pulse with the narrowest width that is multipeaked. Pulses of this shape can still be used in practice, but in some applications the transform-limited pulses are more desirable. These can be achieved with the help of more sophisticated dispersive lines [20-22], even near the resonance points. A choice of parameters that is different from the set shown in Fig. 3 can also improve the pulse chirp and consequently the results for pulse compression. Clearly, more work is needed in this direction.

\section{CONCLUSION}

In conclusion, we have shown that the dissipative soliton resonance observed previously in $[3,4]$ is a robust phenomenon that does not disappear when we strongly perturb the model with parameter management. The phenomenon can be used to generate high energy pulses from a single laser oscillator. The output pulses are usually highly chirped, and the pulse duration can be significantly reduced using a linear dispersive line at the output of the laser system.

\section{ACKNOWLEDGMENTS}

The work was supported by the Australian Research Council (Discovery Project scheme DP0663216). J. M. Soto-Crespo acknowledges support from the Ministerio de Educación y Cultura under contract FIS2006-03376.

\section{REFERENCES}

1. I. S. Aranson and L. Kramer, "The world of the complex Ginzburg-Landau equation," Rev. Mod. Phys. 74, 99-143 (2002).

2. N. Akhmediev and A. Ankiewicz, eds., "Dissipative Solitons," in Lecture Notes in Physics (Springer-Verlag 2005), Vol. 661.

3. N. Akhmediev, J. M. Soto-Crespo, and Ph. Grelu, "Roadmap to ultra-short record high-energy pulses out of laser systems," Phys. Lett. A 372, 3124-3128 (2008).

4. W. Chang, A. Ankiewicz, J. M. Soto-Crespo, and N. Akhmediev, "Dissipative soliton resonances," Phys. Rev. A 78, 023830 (2008).

5. H. A. Haus, "Theory of mode locking with a slow saturable absorber," IEEE J. Quantum Electron. QE-11, 736-746 (1975).

6. W. H. Renninger, A. Chong, and F. W. Wise, "Dissipative solitons in normal-dispersion fiber lasers," Phys. Rev. A 77, 023814 (2008).

7. A. Komarov, H. Leblond, and F. Sanchez, "Quintic complex Ginzburg-Landau model for ring fiber lasers," Phys. Rev. E 72, 025604(R) (2005).

8. H. A. Haus, J. G. Fujimoto, and E. P. Ippen, "Analytic theory of additive pulse mode-locking and Kerr lens mode locking," IEEE J. Quantum Electron. 28, 2086-2096 (1992).

9. P. F. Curley, C. Spielmann, T. Brabec, E. Winter, and F. Krausz, "Periodic pulse evolution in solitary lasers," J. Opt. Soc. Am. B 10, 1025-1028 (1993).

10. S. M. J. Kelly, K. Smith, K. J. Blow, and N. J. Doran, "Averaged soliton dynamics of high-gain erbium fiber laser," Opt. Lett. 16, 1337-1339 (1991).

11. T. M. Fortier, D. J. Jones, and S. T. Cundiff, "Phase stabilization of an octave-spanning Ti:sapphire laser," Opt. Lett. 28, 2198-2200 (2003).

12. J. N. Kutz, B. C. Collings, K. Bergman, S. Tsuda, S. T. Cundiff, and W. H. Knox, "Mode-locking pulse dynamics in a fiber laser with a saturable Bragg reflector," J. Opt. Soc. Am. B 14, 2681-2690 (1997).

13. E. A. DeSouza, C. E. Soccolich, W. Pleibel, R. H. Stolen, M. 
N. Islam, J. R. Simpson, and D. J. DiGiovanni, "Saturable absorber modelocked polarization maintaining erbiumdoped fiber laser," Electron. Lett. 29, 447-449 (1993).

14. F. X. Kartner, L. R. Brovelli, D. Kopf, M. Kamp, I. Calasso, and U. Keller, "Control of solid-state laser dynamics by semiconductor devices," Opt. Eng. (Bellingham) 34, 2024-2036 (1995).

15. F. Krausz, M. E. Fermann, T. Brabec, P. F. Curley, M. Hofer, M. H. Ober, C. Speilmann, E. Wintner, and A. J. Schmit, "Femtosecond solid-state lasers," IEEE J. Quantum Electron. 28, 2097-2122 (1992).

16. G. E. Town and N. Akhmediev, "Optical fiber lasers," in Encyclopedia of Modern Optics, R. D. Guenther, ed. (Academic, 2004), Vol. 2, pp. 475-484.

17. D. Grischkowsky and A. C. Balant, "Optical pulse compression based on enhanced frequency chirping," Appl. Phys. Lett. 41, 1-3 (1982).
18. E. B. Treacy, "Optical pulse compression with diffraction gratings," IEEE J. Quantum Electron. QE-5, 454-458 (1969).

19. G. P. Agrawal, Nonlinear Fiber Optics, 3rd ed. (Academic, 2001).

20. A. M. Weiner, "Femtosecond optical pulse shaping and processing,” Prog. Quantum Electron. 19, 161-238 (1995).

21. T. Katayama and H. Kawaguchi, "Supercontinuum generation and pulse compression in short fibers for optical pulses generated by $1.5 \mu \mathrm{m}$ optical parametric oscillator," Jpn. J. Appl. Phys., Part 2 43, L712-L715 (2004).

22. B. J. Eggleton, G. Lenz, and N. M. Litchinitser, "Optical pulse compression schemes that use nonlinear Bragg gratings," Fiber Integr. Opt. 19, 383-421 (2000). 